\title{
Study of Computerized Segmentation \& Classification Techniques: An Application to Histopathological Imagery
}

\author{
Pranshu Saxena \\ IK Gujral Punjab Technical University, Kapurthala, India \\ E-mail: pranshusaxena@gmail.com \\ Anjali Goyal \\ Guru Nanak Institute of Management and Technology, Ludhiana, India \\ E-mail: anjali.garg73@gmail.com
}

\section{Student paper}

Keywords: computer-assisted diagnosis system, classification, digital pathology, histopathological images, images analysis, segmentation

Received: January 9, 2018

\begin{abstract}
Recent trends with histopathological imagery led to rapid progress towards quantifying the perceptive issues, while prognostic, due to subjective variability among readers. This variability leads to distinguished prognosis reports and generates variability in treatment as well. Latest advancements in image analysis tools have allowed the powerful computer-assisted diagnostic system to assist oncologist in their diagnosis process on radiological data. The main goal of this study is to understand and address the challenges associated with the development of image analysis techniques for computer-aided interpretation of histopathology imagery. We are analyzing indicative characteristics like texture heterogeneity and morphological characteristic on a various scale of lymphomas like Follicular, Neuroblastoma, Breast, and Prostate tissue images for classifying them into respective grades. The study shows a systematic survey of the computational steps, which includes a recent scenario of diagnosis process to classify these lymphomas into respective grades along with its limitations, followed by it shows the pre-requisite of the computer-assisted diagnosis system and finally explains various segmentation techniques based on image descriptor and subsequent classification of biopsy into respective grades. This paper reviews recent state of the art technology for histopathology and briefly describes the recent development in histology and its application towards quantifying the perceptive issue in the domain of histopathology being pursued in the United State and India.
\end{abstract}

Povzetek: Študij tehnik računalniške segmentacije in klasifikacije: uporaba na histopatoloških posnetkih.

\section{Introduction}

In the current scenario, the prognosis of lymphomas is done manually by visual analysis of the tissue samples; obtained from the biopsy of patients. In clinical medicine, a biopsy sample is obtained from a suspicious histological section, placed onto glass slides to be examined under the microscope. Later, biopsy undergoes with staining process preferable Hematoxylin and Eosin (H\&E Stained). H\&E stain is the microscopic study of biological tissues, which will become the gold standard in the prognosis of considerable number of pathologies and for the identification of therapeutic effects. Combination of H\&E stain produces blue, violet and red colors. It provides information about tissues and cells with a high level of detail [1]. Another type of staining known as Immunohistochemical (IHC) staining, is widely used in the diagnosis of abnormal cells and is used to diagnose and track specific cellular anomalies, such as cancers, by identifying those proteins that are specifically found in affected cells. From H\&E Stained images, pathologists inspect morphological characteristic (based on staining) that are indicative of the presence of cancer structures at various scales and determine how closely these structures resemble those in healthy vs. diseased tissues. Sooner the presence of cancer is confirmed, the grading process starts.

The morphological characteristic and texture inhomogeneity of these tissue cells is highly relevant and critically required to predict which patients may be inclined to disease and predicting disease outcome and chances of early survival. This study includes prostate $(\mathrm{CaP})$, breast $(\mathrm{BC})$, neuroblastoma $(\mathrm{NB})$ and follicular lymphoma (FL) tissues histology for critically reviewing the recent state of the art for computer-assisted diagnosis technology, and analyzes the modern state of progress, application of novel image analysis technology and highlights various histopathology related issues.

\subsection{Need for quantitative image analysis for disease grading}

In the current scenario, pathologists play a vital role in examining the digital histopathological image. This examining is done under the microscope but analyzing all 

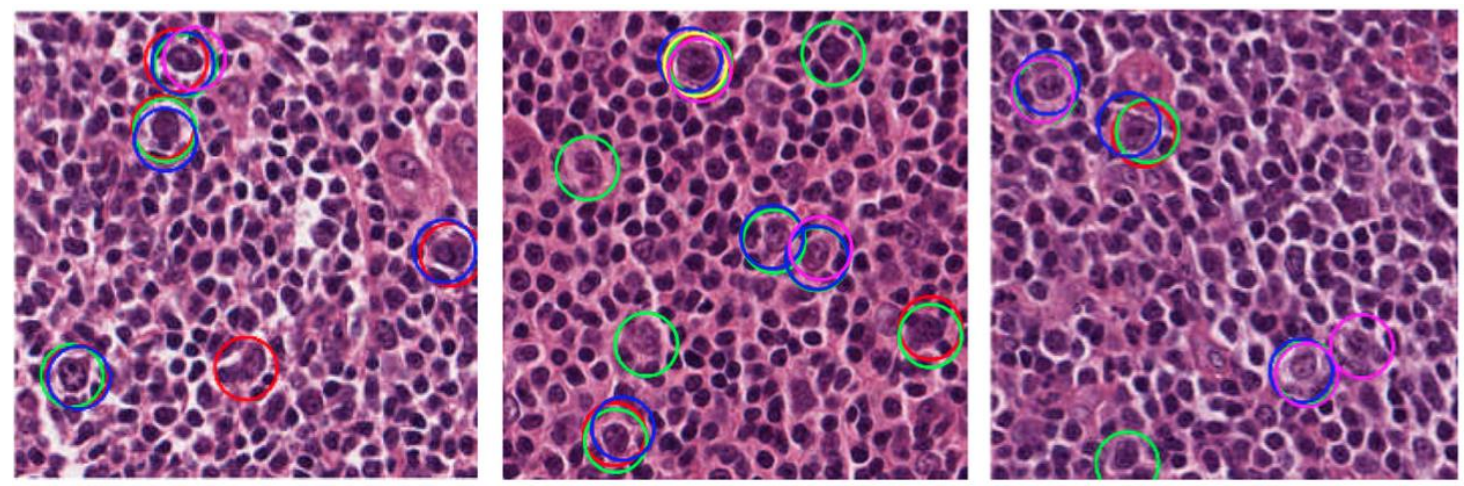

Figure 1: Sample Area of interest taking from $512 \times 512$ pixels H\&E stained FL tissues images; five different oncologists indicating by circles of different colors identify CB cells. [Olcay Sertel et al. 2010] [3].

these microscopic digital images by the pathologist is a very tedious process, and sometimes leads to incorrect conclusions due to several reasons;

- It is not practical to examine every region of the tissue slide under the microscope at high magnifications (e.g., 40×). For cell diagnosis, due to high $\mathrm{CB} / \mathrm{HPF}$ ratio (CB-Centro blast \& HPF- High Power Field i.e. $\left.0.159 \mathrm{~mm}^{\wedge} 2\right)$ in case of FL histology [2].

- The resulting diagnosis can vary considerably among different readers i.e. subjectivity issue.

- Biological variation, heterogeneous intensity, uneven staining, illumination, multiple regions of interest and overlapping the cell nuclei have made prognosis procedure a major impediment.

- There is also an essential requirement for the quantitative-based grading system to ease the workload on pathologists/oncologists by detailed study for non-harmful regions (including all type of cancers); As per a study [1], approximately $80 \%$ of 10 lakh cancer biopsies done every year which results in a negative response. This implies that there was, no need to do the biopsy at all.

Moreover, quantitative analysis can be very useful for research application like drug discovery, biological mechanisms in disease, identifying the pattern of genetic abnormalities in cancerous nuclei. But recent research says, [39] analyzing transformation in biological tissues remains a challenge due to unavailability of the robust biomarker in routine clinical practice. Indeed, several genetic alterations, IHC stained or H\&E stained markers have been reported in previous years associated with biological transformation but haven't been sufficiently validated to warrant their assessment outside of the research setting. Use of computer-assisted diagnosis in pathology can substantially enhance the efficiency and accuracy of pathologists in decision-making, which indeed favors the patients [1]. It is emphasized that the automated computer-assisted diagnosis system should never be considered a replacement for oncologists. Instead, it should only be used as assistance to the decision-making mechanism. In case of a disagreement between the computerized system and the oncologists rating, the final decision is that of the human doctors i.e. oncologists.

With the recent trends and advancement in the CAD system, this review discusses and depict different methods suggested in the literature for segmentation and subsequent classification of $\mathrm{FL}, \mathrm{BC}, \mathrm{NB}, \& \mathrm{CaP}$ histopathological images. The main emphasis of identifying the most often methods of lymphoma images segmentation, such as thresholding, fourier based transformation, region-based segmentation, k-means clustering, statistical shape model, texture-based segmentation, and methods for their classification into respective grades, such as supervised and unsupervised clustering, laplacian eigen-map classifiers, k-nearest neighbor, rule-based classifiers and neuro-fuzzy inference system. The major contribution of this study is found in the discussion of the main processing techniques of lymphoma histological images, therefore, providing directions for future research.

\subsection{Organization of this paper}

We have organized this paper to follow the general image analysis procedure for histopathological imagery. These analysis procedures are usually applicable to all histopathological imagery. In section 2, we present the definition and details about quantitative criteria for disease grading on the various scale for $\mathrm{FL}, \mathrm{NB}, \mathrm{CaP}$, and $\mathrm{BC}$. In section 3, we have reviewed various histological slide preparation process followed by image pre-processing steps such as colour normalization, image representation. Later, a systematic review is placed for segmentation and classification for various lymphomas followed by inference from the review of literature in the table. Discussion and future directions for research of lymphomas image segmentation considering limitation for published articles, followed by the conclusive remark is presented in Section 4.

\section{Quantitative criteria for disease grading}

In the current scenario prognosis of the diseases such as $\mathrm{CaP}, \mathrm{BC}, \mathrm{FL} \& \mathrm{NB}$ is done manually by visual analysis of the tissue samples, obtained from the biopsy of patients. This visual grading is very tedious and sometimes leads to under and over treatment due to inter-reader and intrareader variability; this result indicates incapable situation for the patient, figure 1 shows distinguished prognosis report of the same biopsy of FL sample. In Figure 1, the 


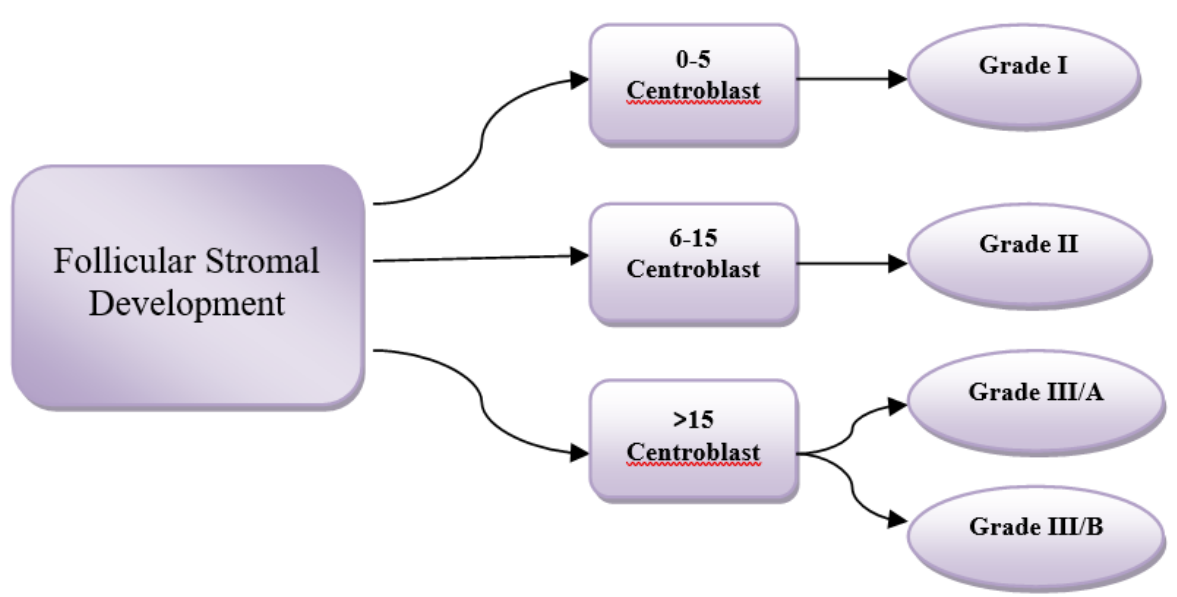

Figure 2: WHO classification of FL based on centroblast/HPF; Grade I \& Grade II are considered as low-risk categories, while grade III belongs to high categories.

prognosis process is explained using three different follicular tissue samples by 5 distinguished oncologists. The oncologists were asked to locate the area of interest. All five oncologists have marked their perceptive views with different colors on the tissue sample, which clearly shows a different perceptive view with different oncologists.

A computer-assisted diagnosis system for digitized histopathology is pre-requisite that is employed for disease prognostics, allowing the oncologist to predict which patients may be susceptible to disease and predicting disease outcome and chances of survival. Automated image segmentation of cell nuclei and subsequent classification of histopathological images is widely research topic. Several distinguished researchers have been developing the algorithms for the automated segmentation and classification. There have been few attempts to automatically detect the grading and to newer methods continue to be investigated. The need for quantitative image analysis in the context of some specific diseases (like prostate, breast, follicular lymphoma \& neuroblastoma) is described in the next section.

\subsection{Grading criteria for follicular lymphoma by WHO}

The World Health Organization (WHO) recommends Histopathological grading of Follicular lymphoma based on the number of large malignant cells, namely $\mathrm{CB}$, per standard 40x magnifications High Power Field (HPF) of $0.159 \llbracket \mathrm{mm} \rrbracket \wedge 2$. In this method, CB's are manually counted and the average of $\mathrm{CB} / \mathrm{HPF}$ is reported $[2,4]$.

According to WHO, high power field of $\mathrm{H} \& \mathrm{E}$ stained tissue sections (under the microscope) classify the biopsy into one of the three histopathological grades according to the average $\mathrm{CB}$ count per HPF, this grading criterion is plotted in the figure 2 . In grades, I and II, the proportion of small cell (centrocytes) is predominant, whereas grade III features a greater proportion of large cells (centroblast). The histopathological examination guides the oncologists in making timely decisions and on the required therapy [2, 4-22]. This clinical relevance of grading system is still being debatable [38]; Grade I, II, IIIA are sluggish and incurable, while Grade IIIB belongs to most aggressive but curable. In the above classification, Grades I and II belong to quantitative issue (count the number of $\mathrm{CB} / \mathrm{HPF}$ ) while classification of Grade III in its respective subgroups requires qualitative issue as $\mathrm{CB} / \mathrm{HPF}$ ratio remains same ( $>15$ in both cases Grade IIIA \&B). The only subjective issue which distinguishes between Grade IIIA \& B is that grade IIIA shows the presence of centrocytes, whereas in IIIB the follicles consist almost entirely of the centroblastic cell. Although patients suffering from indolent FL (Grade I, II \& IIIA) typically live for many decades with minimal or no treatment, while patients with aggressive FL (IIIB) have short survival if not treated appropriately at early stages.

\subsection{Grading criteria for neuroblastoma by WHO}

Neuroblastoma is the most common extracranial solid cancer, commonly affects to infant and children (0-5 years). Based on the American Cancer Society [2] statistics, it is by far the most common cancer in infants and the third most common type of cancer in children. WHO recommends the use of the International Neuroblastoma Pathology Classification (the Shimada system) for categorization of the patients into different prognostic stages. This classification system is based on morphological characteristics of the tissue.

Figure 3 shows a relevant summary of this classification system as a tree diagram. Shimada system includes the age of patients, the degree of neuroblast differentiation, presence or absence of schwannian stormal development, mitosis and karyorrhexis index (MKI) and nodular pattern to conclude the final tissue classification as favorable histology (FH) and unfavorable histology (UH) [4, 23]. MKI index is calculated based on the number of Karyorrhectic cells per number of cells scanned in the sample (200 Karyorrhectic cells for every 5000 cells scanned).

Although the shimada system performs fine analysis in $80 \%$ cases and provides a precise decision, it may be disingenuous for heterogeneous tumors. A study by Teot et al. in 2007 [24] shows that for NB diagnosis, this 


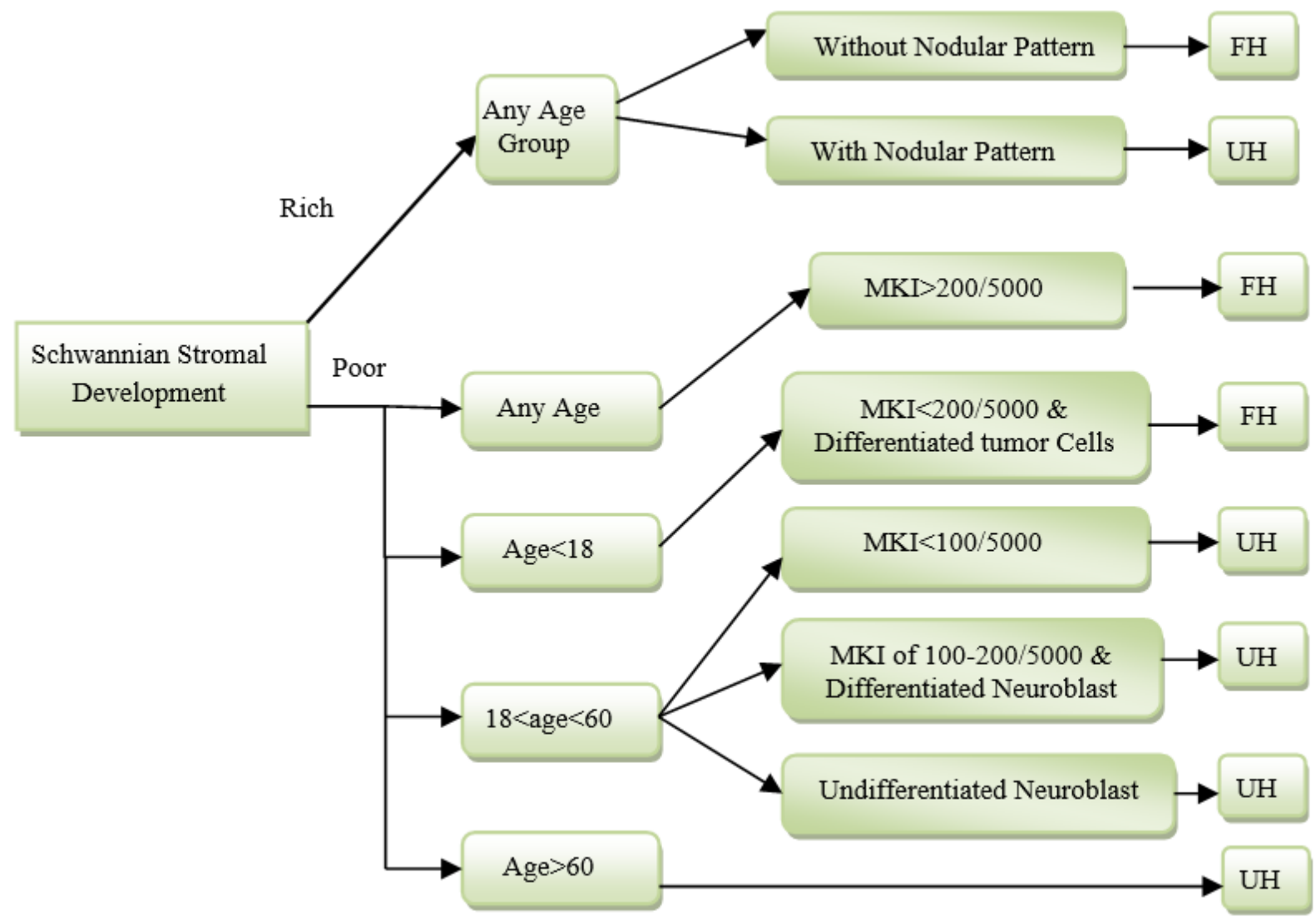

Figure 3: Shimada classification of neuroblastoma tissue sample into favorable \&unfavorable histology based on schwannian stromal development.

variation can be up to $20 \%$ between central and institutional reviewers.

\subsection{Gleason scale for prostate cancer}

Quantitative image analysis in the context of prostate cancer is done with the help of gleason score [25], persons having high gleason score are more aggressive and have a worse prognosis. Grading is solely based on the morphological pattern of tissue samples. Dr. Gleason shows 1 to 5 histological patterns of decreasing differentiation (pattern-1 most differentiated \& pattern-5 least differentiated). Based on this score, doctors predict the stage of the disease.

C. R. King et al. in 2000 [26] have found grading error (both under \& over Grading) in prostate histology. Additionally, the accuracy of the classification is important to prevent making any under or over treatment. Unfortunately, this classification becomes more vulnerable when oncologist's prognostic these highly microscopic (e.g. 40x) biopsies by only picking a small representative section (e.g. $2 \mathrm{x}$ or $4 \mathrm{x}$ ) and concludes for a whole slide (40x) tissue sample.

\subsection{Nottingham histologic score system for breast histology}

For classification of breast histology, Bloom \& Richardson introduced a system in 1957 based on cellular differentiation [27]. Later, Elston-Ellis in 1991 introduced Nottingham Histologic score system grounded on three criterions: 1) How well the cancerous cell tries to recreate normal glands (amount of gland formation); 2)How ugly the cancerous cell looks (nucleolus features); 3) How much the cancerous cells are divided (mitotic activity). Pathologist gives the score to each of these criterions in 13 scale, and each score is added to give final total score 3 9 , this final score is responsible to classify breast biopsy into 3 criterions; [28]

- Grade 1 tumors have a score of 3-5 (well differentiated, slow growing).

- Grade 2 tumors have a score of 6-7 (moderate differentiated).

- Grade 3 tumors have a score of 8-9 (poorly differentiated, highly proliferative).

New genetic re-classification of histological grades is introduced by Anna V. Ivshina et al. in 2005 due interobserver variability among pathologists while classifying biopsy into either grade 2 or grade 3. [29]

\section{Study to computer-assisted diagnosis system}

\subsection{Staining process of biopsy}

In the current scenario, histopathological tissue analysis is done manually by visual analysis of tissue sample, obtained from the tissue biopsy of the patients. In clinical medicine, a biopsy sample is obtained from a suspicious histological section, placed onto glass slides to be examined under the microscope. Before microscopy 
examination biopsy follows a series of procedure. First, step named as a fixation step, that aims to preserve the morphological and architectural structure of the sample followed by a procedure named as embedding that allows slicing the biopsy in very thin likely $2-15 \mu \mathrm{m}$ area section. Finally, this slice goes to the staining process.

Over the several staining presents in the current scenario, Hematoxylin and Eosin (H\&E stain) is one of the principles one. It is most widely used staining medical science. A combination of hematoxylin and eosin dyes produces blues, violets, and red colors to demonstrate nucleolus \& cytoplasmic. It provides information about tissues and cells with a high level of detail [1]. The stain has been unchanged for many years because it works well with the variety of fixatives and displays a broad range of cytoplasmic, nuclear and extracellular matrix feature.

Another type of staining known as Immuno-HistoChemical (IHC) staining, is widely used in the diagnosis of abnormal cells and is used to diagnose and track specific cellular anomalies, such as cancers, by identifying those proteins that are specifically found in affected cells. In clinical practice, IHC stain is used to mark the follicle regions where B-cell are positively marked with hues of brown color as opposed to T-cell that is negatively marked with hues of gray color. Therefore, follicles, which have a higher concentration of B-cell, can be distinguished from inter-follicular regions at low magnifications (i.e., $2 \times, 4 \times, 8 \times)$.

\subsection{Image preprocessing: color normalization}

After the staining process, color normalization is very essential preprocessing step for microscopy image. This process reduces the differences in tissue samples due to variation staining conditions. Moreover, this step is to remove irrelevant tissue structures, remove noises, and enhance the contrast. Primarily preprocessing techniques aim to improve image quality for segmentation.

Color normalization step is very commonly used to lymphoma images processing. The technique helps to provide different perceptual difference among color models $[8,9,11]$. O. sertel et al. [8, 9] used the conversion from the RGB model to $\mathrm{L}^{*} \mathrm{a}^{*} \mathrm{~b}^{*}$, Euclidean distance is the measure to exploit the differences while [11] used the conversion from the RGB model to the HSV model. These color models help to segmentation process by representing each cytological component with different colors. In [3] $\mathrm{RGB}$ color space is projected to a 1-D uni-tone image by considering only 1 st PCA, to attain a single channel image that has the highest contrast.

\subsection{Automated segmentation and classification of histopathological image}

Use of CAD in medicine is drastically increasing day by day, to aid in detection, diagnosis of disease in digital pathology. To fulfill the requirement of the system, researchers identify certain quality parameters of images such as the morphological structure of lymphocytes, cancerous nuclei, and glands. The presence of extent shape, size and other morphological appearance of these structures are important indicators for the presence of severity of the disease. For instance, for neuroblastoma histology, the existence of a nodular pattern and distinguish and un-distinguish neuroblast leads to favorable and unfavorable histology. In terms of prostate lymphoma, the size of glands tends to reduce with higher Gleason patterns. Similarly, the presence of many lymphocytes in breast cancer histopathology is strongly suggestive of poor disease outcome and survival.

Another motivation for detecting and segmenting histological structures has to do with the need for counting of objects, generally cells or cell nuclei. Cell counts can have diagnostic significance for some cancerous conditions. For instance, in terms of FL, pathologist counts the number of CBs per HPF also in terms of NB MKI index is calculated based on the number of Karyorrhectic cells per number of cells scanned in the sample. Bibbo et al. [30] reported $1.1 \%-4.7 \%$ error in cell counts compared to manual counts for Feulgen-stained prostate specimens. In order to evaluate segmentation methods, several matrices have been proposed for evaluating computational and manual segmentation conducted by the oncologist. Among these matrices, common names sensitivity, specificity, accuracy considering the concept of true positive, false positive [15]. Accuracy can quantify how many pixels from manual segmentation were also identified by the computational methods [15]. Another metric named Zijdenbos similarity index [11], This measures the overlapping ration between the shapes from manual and automatic segmentation. The systematic survey is presented for diagnosing and prognosis techniques using computational techniques.

A very basic CAD model started in 2007, to aid pathologists in prognosis for histological grading system of FL histology by J. Kong et al. [5] in which pathologist's (human) intervention was also required along with computer-assisted diagnosis model in the classification of FL. Firstly, color and texture are features extracted to detect follicles from H\&E stained images using K-means clustering method, followed by a morphologic postprocessing step to remove the noisy regions and smooth out the boundaries of follicle regions. Subsequently, a manual registration step is performed to detect distorted of follicles and healthy tissue sample and finally classification process proceeds to group data into $\mathrm{CB}$ and non-CB classes. In continuation of previous work [5], O. Sertel et al. in 2008 [6] develops a computerized system to reduce manual intervention based on non-supervised clustering to assist pathologists in differentiating CB from non-CB cells. Comparative analysis between similarity index between computerized system \& pathologist is discussed in table 1 . Till now researchers have been only attempting to differentiate between $\mathrm{CB}$ and non-CB cells $[5,6]$, but in 2008, O. Sertel et al. [7] introduced new classification index based on WHO criteria. The WHO recommends histopathological grading of FL based on the number of $\mathrm{CB}$, per standard 40x magnifications HPF of $0.159 \mathrm{~mm}^{\wedge} 2$ [4]. To capture this information, firstly images 
were partition into distinct cytological components (nuclei, cytoplasm, RBC, background and extracellular material) based on unsupervised segmentation technique followed by color texture is extracted by non-linear quantization using self-organizing map, which is used to differentiate the low and intermediate grades (I and II) from high grades (III) of FL. For the classification of the segmented image (from the first step), principle component analysis (PCA) and linear discriminant analysis (LDA) followed by Bayesian parameter estimation are used and again O. Sertel et al. in 2009 [8], introduced a novel computer-assisted diagnosis system for digitalized histology based on model-based intermediate representation (MBIR) and incorporates texture analysis. The system identifies basic cytological component using La*b* color space if the same amount of change in color values produces the same amount of perceptual difference of visual importance. Property of $\mathrm{La}^{*} \mathrm{~b}^{*}$ color space allows to use euclidean distance in comparing colors. Clustering in $\mathrm{La}^{*} \mathrm{~b}^{*}$ color space is done using the K-means algorithm. Researcher uses PCA and FLDA to reduce the dimension, followed by bayesian classifier based on maximum a posterior decision rule for classification.

Limitations of conventional feature space-based clustering algorithm like K-mean and EM (expectation Minimization) are identified by O. Sertel et al. in 2010 [9], those are widely used for Histopathology image segmentation $[5,6,7,8]$. Those algorithms based on prior knowledge of clusters and confined to the mostly elliptical shape of these clusters. In fact, especially in histopathology imagery, these assumptions may not suit well due to inhomogeneous intensities and overlapping cell nuclei. In order to achieve a robust approach, which can overcome the limitation of clustering-based feature space; a non-parametric method is introduced [9], namely the mean-shift method. This method is applied to L*a*b* color space and finds out stationary points of density. Adaptive thresholding technique is used to differentiate between $\mathrm{CB}$ and non-CB cells. In fact, this is a very initial detection system based on the non-parametric approach to distinguish $\mathrm{CB}$ and non-CB cells with relatively very high false positive [9].

To overcome these very high false positives is being experienced by [9], O. Sertel et al. in 2010 [3] introduces a better approach grounded on adaptive likelihood-based cell segmentation. This approach includes, Firstly, input images in the RGB color space is projected onto a 1-D unitone image by considering only first principal component, to attain a single channel image that has the highest contrast. This uni-tone image is further normalized between $[0,1]$. Subsequently, two steps process is applied for the classification of $\mathrm{CB}$ and non-CB cells as follows.

- Identify evident non-CB cells based on size, shape, and eccentricity.

- Redefine $\mathrm{CB}$ detection by learning and utilizing the texture distribution of non-CB cells.

In 2010, Siddhartha Samsi et al. [10] described an automated system to identify follicles in IHC stained tissue section rather than H\&E stained images. IHC stains are typically used to identify specific categories of cells in the tissue. The proposed algorithm uses color and texture measures for identifying follicles and their respective boundarization. To reduce under and over-segmentation, the author applied the watershed segmentation algorithm and finally, fourier descriptors are used to smooth follicle boundary.

A modified paper of [3] is introduced to improve the accuracy of pathologists by extracting the morphological characteristic of objects based on novel texture features, color-space decomposition. Instead of grayscale representation of images, color-space is utilized, and RGB, L*a*b*, HSV color spaces are investigated. K. Belkacem-Boussaid et al. in 2010 [11] uses a multivariate image analysis technique using PCA to classify between $\mathrm{CB}$ and non- $\mathrm{CB}$ tissue samples. For extraction of the geometry of $\mathrm{CB}$, researchers use operations such as thresholding, morphological filtering, and area identification.

Again, the different approach introduced by $\mathrm{K}$. Belkacem-Boussaid et al. in 2011 [12] in which, follicular regions are identified first at lower magnification (2X). Later, higher magnification is used to identify and count the number of centroblastic cells. Three step procedures are applied to start form region-based segmentation, which is used to determine the location of the follicle regions, followed by iterative shape index calculation and finally recursive watershed algorithm is applied. For initial segmentation of follicles, Chan \& Vese [13] introduced a region-based segmentation approach based on curve expansion. With respect to the interior of follicles, the curve starts moving, and energy of curve minimized where the desired boundary is achieved. During boundarization process, level set formulation leads to very closed objects (likely overlapped) to each other; hence level set formulation is venerable to overlapping combined follicles. To overcome these overlapping follicles problems, an adaptive control splitting and merging technique is applied at the object level. The study presents a control factor based on the concavity index for the individual object. Followed by a novel recursive marked-watershed operation. Combining all the operation produces a better segmentation for overlapped follicles and hence prevents limitations that usually are introduced during the traditional morphological watershed is overcome. A combined effort to reduce under and oversegmentation of overlapped cells is made by H. Kong et al. in 2011 [14]. This framework firstly segments the histopathological images based on the local context features extracted around the pixel. In which, each pixel is categorized into either the cell or extracellular classes. After that local Fourier transformation is applied for extracting the texture feature based on newly defined color space, called the most discriminant color space (MDC).

A different approach to isolating FL tissue samples, M. Oger et al. in 2012 [15] presented a noble technique. In this technique segmentation of follicular areas is recognized firstly before histological grading is done. Following the current practice of the pathologist, low resolution, lower magnification (2X) IHC Stained image uses to generate a mask of the follicular boundaries (follicular area) by a newly deployed feature-based cluster approach. Then these boundaries are map onto a 
corresponding registered H\&E stained image. From these $\mathrm{H} \& \mathrm{E}$ stained images, color and texture information is extracted by converting the image from RGB space to HSV space. Where S (saturation) channel is represented as color information. Texture information is quantified by the homogeneity of $9 \times 9$ neighborhoods of each pixel using the co-occurrence matrix approach. Resulting feature vector is classified using k-means classifiers with $\mathrm{K}=4$ one for follicles, second for the intra-follicular area, third for the mixture of follicles and intra-follicular area and last for the background. With respect to previous researches $[10,15]$, an efficient computational framework has been proposed [16] for the analysis of whole slide images (an application to FL immunohistochemistry stain) by S. Samsi et al. in 2012. This framework involves calculation of color and grayscale features, which are used as feature vectors for $\mathrm{K}$-means clustering. The color feature used is the hue channel from HSV color-space conversion of the original image. The first texture feature used is the output of a median filter of size $45 \square 45$ applied to a gray-scale version of the image. The second texture feature is the energy feature calculated from the cooccurrence matrix. The output of the clustering algorithm provides a segmented image that is further processed by an iterative watershed algorithm followed by a boundarysmoothing step.

B. Oztan et al. in 2012 [17] introduced a new computer-aided technique for grading of FL tissue sample based on cell graph and multi-scale feature analysis. FL image is analyzed using the cell graph to know the structural organization of component like nuclei, cytoplasm. Cell graph technique includes a graph theory concept to represent FL image with the un-weighted and undirected graph. Nodes of the graph are represented as cell nuclei whereas adjacencies of cells are represented with the edges of the graph. After constructing the cellgraph representation, a feature space vector is formulated, which includes structural organization within the nuclei and cytoplasm components.

E. Michail et al. in 2014 [18] projected a new scheme for detection of $\mathrm{CB}$ from $\mathrm{H} \& \mathrm{E}$ Stained images. Detection of $\mathrm{CB}$ starts from converting the image into grayscale and filter using the Gaussian filter with kernel $3 \square 3$. Moreover, in order to detect the nuclei, the difference between nuclear membrane and background are enriched by histogram equalization technique. Later, segmentation of nuclei (dark) from extracellular material and background, otsu-thresholding is applied. Additionally, Expectation maximization (EM) technique is used to separate touching cells. Isolation is CBs is done based on shape, size, and intensity of histogram criteria. Finally, LDA is used to classify CBs and non-CBs cells.

E. N. kornaropoulosin et al. in 2014 [19] introduced a quantitative methodology to categorize FL histology into one of two categories of cells (CB vs non-CB) using linear and non-linear dimensionality reduction. In the first method, biased features are calculated with the help of singular value decomposition, in which discrimination between $\mathrm{CB}$ and non-CB problem can be formulated as a minimization problem, where the objective is to minimize the least-square error between given images of $\mathrm{CB}$ and
non-CB its low-rank approximation. In the second method, the classifier is based on preserving the similarity among the images of $\mathrm{CB}$ and non- $\mathrm{CB}$ cells using Laplacian eigen-maps.

K. Dimitropoulos et al. [20] in 2014 presented a study for automatic detection of CBs from microscopic images obtained from FL biopsy. Initially, the touching-cell splitting algorithm is applied using the Gaussian mixture model and EM algorithm to segment the biopsy into basic cytological elements. Additionally, morphological and textural analysis of $\mathrm{CBs}$ is applied to extract various features related to the nucleolus, cytoplasm extra-cellular cells. In the final step, an innovative classification scheme is proposed based on adaptive neuro-fuzzy inference system to classify the interested cells.

Follicular lymphoma grading system, a color-coded map-based system was presented by M. Faizal Ahmad Fauzi et al. in 2015 [21]. The system includes the HSV color model to register the two images to obtain a good gray level separation among the follicle regions, nonfollicle regions, and the white background. Classification in respective grades has been done using $\mathrm{KNN}$ classifier of potential $\mathrm{CB}$ regions within sub-blocks of the HPF regions followed by rule-based classification at the block, HPF and tissue levels.

A novel framework is proposed by K. Dimitropoulos et al. in 2016 [22] for segmentation, separation, and classification of CBs and Non-CBs cells from $\mathrm{H} \& \mathrm{E}$ and IHC Stained FL histology. For segmentation of nuclei, energy minimization technique based on the graph-cut method is applied to IHC Stained images. A noble algorithm based on inspired by clustering of large-scale visual terms is used to segment the nuclei. Additionally, H\&E stained images enable to extract textural information related to histological characteristics. Finally, morphological characteristic from IHC staining and textural information from H\&E Stained Images are used to construct the feature vector. This feature vector is then fed into Bayesian network classifier to classify FL histology into respective grades.

In 2012, Scott Doyle et al. [31] (2012) headed a classification technique for Prostate histology. In the first step, this algorithm decomposes the whole-slide image into an image pyramid comprising multiple resolution levels. Regions identified as cancer via a Bayesian classifier at lower resolution levels are Subsequently examined in greater detail at higher resolution levels, thereby allowing for rapid and efficient analysis of large images. Later, Sahirzeeshan Ali et al. [32] in 2012, commented and enhanced classification accuracy by demonstrating a robust algorithm, which provides boundaries close to the actual nuclear boundary and the shape constraint prevents spurious edges. The algorithm also demonstrates an application of these synergistic active contour models using multiple level sets to segment nuclear and glandular structures on digitized histopathology images of breast and prostate biopsy specimens.

A Manifold learning (ML) scheme is presented by Rachel Sparks et al. [33] in 2013, which attempts to generate a low dimensional manifold representation of a 
higher dimensional feature space while simultaneously preserving nonlinear relationships between object instances. Classification can then be performed in the low dimensional space with high accuracy while, Safa'a N. AlHaj Saleh et al. [34] in 2013 uses K-means clustering based approach was employed to the $\mathrm{a}^{*}$ color channel of the $\mathrm{L}^{*} \mathrm{a} \mathrm{b}^{*}$ color model for each of the tissue images, followed by statistical and morphological features extraction for the segmented lumen objects and glands. Finally, a naive bayes classifier was used to classify tissue images to the correct grade. In 2016, M Khalid Khan Niazi et al. [35] provides additional view angle meaningful representation and discriminates between low and high graded prostate lymphoma. These meaningful representation features include luminal and architecture feature. These features help to create two subspaces one for prostate histology assessed as a low grade and other to classify histology into the high grade.

An idea to work with gray tone images, which are represented by two descriptors, one is local binary pattern $\&$ other is local phase quantization, a noble approach is presented by Ville Ojansivu et al. [36] in 2013. The classification of the images into the three classes was done using three one-versus-rest SVM classifiers with a radial basis function kernel (RBF) combined with a chi-square distance metric. Selecting the largest of the scores produced by the individual SVM classifiers chose the final class. Additionally, Maqlin Paramanandam et al. [37] in 2016 propose a novel segmentation algorithm for detecting individual nuclei from Hematoxylin and Eosin (H\&E) stained breast histopathology images. This detection framework estimates a nuclei saliency map using tensor voting followed by boundary extraction of the nuclei on the saliency map using a loopy back propagation algorithm on a markov random field. The algorithm uses hough transformation to identify seeds points, which are used to initializing shape- and texture based active contour algorithm.

In 2009 O. Sertel et al. [23] Paper, introduced a segmentation technique grounded on texture feature, which is extracted using local binary pattern and cooccurrence. This statistical framework uses modified knearest neighbor classifier is used to determine the confidence level of the classification to make the decision at a resolution level.

\begin{tabular}{|c|c|c|c|c|c|}
\hline $\begin{array}{l}\text { Segmentation \& } \\
\text { Classification } \\
\text { Techniques }\end{array}$ & $\begin{array}{l}\text { Refer } \\
\text { ence }\end{array}$ & $\begin{array}{l}\text { Public } \\
\text { ation } \\
\text { year }\end{array}$ & $\begin{array}{l}\text { Imag } \\
\text { es } \\
\text { types }\end{array}$ & Object of Interest & Evaluation result \\
\hline $\begin{array}{l}\text { K-means clustering } \\
\text { followed by manual } \\
\text { registration }\end{array}$ & {$[5]$} & 2007 & FL & $\begin{array}{l}\text { CB \& non-CB cells } \\
\text { classification }\end{array}$ & $\begin{array}{l}\text { There is no performance } \\
\text { evaluation for } \\
\text { classification }\end{array}$ \\
\hline $\begin{array}{l}\text { Non-supervised } \\
\text { clustering }\end{array}$ & {$[6]$} & 2008 & FL & $\begin{array}{l}\text { CB \& non-CB cells } \\
\text { classification }\end{array}$ & $\begin{array}{c}\text { Maximum accuracy of } \\
90.7\end{array}$ \\
\hline $\begin{array}{c}\text { Non-linear } \\
\text { quantization using } \\
\text { SOM followed by LDA } \\
\text { \& BPE }\end{array}$ & {$[7]$} & 2008 & FL & CBs per HPF calculation & $\begin{array}{l}88.9 \% \text { accuracy of FL } \\
\text { tissue classification }\end{array}$ \\
\hline MBIR & {$[8]$} & 2009 & FL & CBs per HPF calculation & $\begin{array}{l}88.7 \% \text { correctness } \\
\text { achieved }\end{array}$ \\
\hline $\begin{array}{c}\text { Texture \& color-based } \\
\text { Classification using } \\
\text { KNN }\end{array}$ & {$[23]$} & 2009 & NB & $\begin{array}{l}\text { Identification of Stroma rich } \\
\text { and stroma poor }\end{array}$ & $\begin{array}{l}\text { overall classification } \\
\text { accuracy of } 88.4 \% \text {. }\end{array}$ \\
\hline Mean shift method & {$[9]$} & 2010 & FL & $\begin{array}{l}\text { CB \& non-CB cells } \\
\text { classification }\end{array}$ & $\begin{array}{l}\text { qualitative very high false } \\
\text { positive appealed }\end{array}$ \\
\hline $\begin{array}{l}\text { Adaptive Likelihood- } \\
\text { based cell segmentation }\end{array}$ & {$[3]$} & 2010 & FL & $\begin{array}{l}\mathrm{CB} \& \text { non-CB cells } \\
\text { classification }\end{array}$ & $\begin{array}{c}\text { maximum accuracy } 80.7 \% \\
\text { achieved }\end{array}$ \\
\hline $\begin{array}{l}\text { Watershed with } \\
\text { Fourier descriptors }\end{array}$ & {$[10]$} & 2010 & FL & Follicular Region & $\begin{array}{l}87.11 \% \text { accuracy while } \\
\text { classifying follicles }\end{array}$ \\
\hline $\begin{array}{l}\text { Multivariate image } \\
\text { analysis using PCA }\end{array}$ & {$[11]$} & 2010 & FL & $\begin{array}{l}\text { CB \& non-CB cells } \\
\text { classification }\end{array}$ & $\begin{array}{l}82.57 \% \text { precision while } \\
\text { segmenting }\end{array}$ \\
\hline $\begin{array}{c}\text { Fourier } \\
\text { transformation, most } \\
\text { discriminant color } \\
\text { space }\end{array}$ & {$[14]$} & 2011 & FL & Cellular nuclei & $\begin{array}{l}\text { The total error rate is } \\
5.25 \% \text { per image }\end{array}$ \\
\hline $\begin{array}{l}\text { Region based } \\
\text { segmentation followed } \\
\text { by the iterative index }\end{array}$ & {$[12]$} & 2011 & FL & Follicular Region & $\begin{array}{c}\text { There is no performance } \\
\text { evaluation for } \\
\text { classification }\end{array}$ \\
\hline
\end{tabular}




\begin{tabular}{|c|c|c|c|c|c|}
\hline $\begin{array}{l}\text { and finally recursive } \\
\text { watershed }\end{array}$ & & & & & \\
\hline $\begin{array}{l}\text { Region-based active } \\
\text { contours with the } \\
\text { statistical shape model }\end{array}$ & [32] & 2012 & $\begin{array}{l}\mathrm{CaP} \\
\& \mathrm{BC}\end{array}$ & $\begin{array}{l}\text { Segment overlapping } \\
\text { lymphocytes and lumen }\end{array}$ & $\begin{array}{l}90 \% \text { accuracy while } \\
\text { segmentation }\end{array}$ \\
\hline $\begin{array}{l}\text { Boosted Multi- } \\
\text { resolution Classifier }\end{array}$ & [31] & 2012 & $\mathrm{CaP}$ & Regions of $\mathrm{CaP}$ & $\begin{array}{c}\text { There is no performance } \\
\text { evaluation for } \\
\text { classification }\end{array}$ \\
\hline K-Mean Classifier & {$[15]$} & 2012 & FL & $\begin{array}{l}\text { The follicular area along with } \\
\text { Follicles detection }\end{array}$ & $\begin{array}{c}\text { There is no performance } \\
\text { evaluation for } \\
\text { classification }\end{array}$ \\
\hline $\begin{array}{l}\text { K-mean, co-occurrence } \\
\text { matrix followed by } \\
\text { watershed \& boundary } \\
\text { smoothing }\end{array}$ & {$[16]$} & 2012 & FL & Identification of follicles & $\begin{array}{c}\text { There is no performance } \\
\text { evaluation for } \\
\text { classification }\end{array}$ \\
\hline $\begin{array}{l}\text { Cell graph followed by } \\
\text { supervised learning }\end{array}$ & {$[17]$} & 2012 & FL & $\begin{array}{l}\text { Detection of nuclei and other } \\
\text { cytological components }\end{array}$ & $\begin{array}{l}87 \% \text { accuracy in } \\
\text { classification }\end{array}$ \\
\hline $\begin{array}{l}\text { A Statistical Shape } \\
\text { model of manifolds }\end{array}$ & [33] & 2013 & $\mathrm{CaP}$ & Manifold regulization & $\begin{array}{c}\text { There is no performance } \\
\text { evaluation for } \\
\text { classification }\end{array}$ \\
\hline $\begin{array}{l}\text { Texture Based } \\
\text { Classification }\end{array}$ & {$[36]$} & 2013 & $\mathrm{BC}$ & Breast glands & $\begin{array}{l}\text { This work achieves the } \\
\text { classification accuracy of } \\
\text { breast histology is } 90 \% \text {. }\end{array}$ \\
\hline $\begin{array}{l}\mathrm{L} * a * b *-b a s e d \\
\text { segmentation }\end{array}$ & {$[34]$} & 2013 & $\mathrm{CaP}$ & $\begin{array}{l}\text { Lumen objects and tissue } \\
\text { glands }\end{array}$ & $\begin{array}{l}\text { Automated classification } \\
\text { results achieved the } \\
\text { accuracy of } 91.66 \% \text {. }\end{array}$ \\
\hline $\begin{array}{l}\text { Thresholding for } \mathrm{RBC} \\
\text { removal and Otsu to } \\
\text { segment nuclei \& LDA }\end{array}$ & {$[18]$} & 2014 & FL & Centro blast detection & $\begin{array}{c}82.58 \% \text { CB were } \\
\text { successfully detected }\end{array}$ \\
\hline $\begin{array}{l}\text { Linear and non-linear } \\
\text { dimensionality } \\
\text { reduction followed by } \\
\text { Laplacian Eigen map } \\
\text { classifier }\end{array}$ & [19] & 2014 & FL & $\begin{array}{l}\text { CB \& non-CB cells } \\
\text { classification }\end{array}$ & $97.67 \%$ of accuracy \\
\hline $\begin{array}{l}\text { Linear and non-linear } \\
\text { dimensionality } \\
\text { reduction followed by } \\
\text { orthogonal bases }\end{array}$ & [19] & 2014 & FL & $\begin{array}{l}\text { CB \& non-CB cells } \\
\text { classification }\end{array}$ & $98.22 \%$ of accuracy \\
\hline $\begin{array}{l}\text { Touching cell splitting } \\
\text { using GMM followed } \\
\text { by neuro fuzzy } \\
\text { inference system }\end{array}$ & {$[20]$} & 2014 & FL & $\begin{array}{l}\text { Cytoplasmic element } \\
\text { classification }\end{array}$ & $\begin{array}{l}90.35 \% \text { detection rate } \\
\text { achieved }\end{array}$ \\
\hline $\begin{array}{c}\text { Color coded map- } \\
\text { based segmentation } \\
\text { followed by rule-based } \\
\text { classification }\end{array}$ & [21] & 2015 & FL & CBs per HPF calculation & $80 \%$ correct classification \\
\hline $\begin{array}{l}\text { Texture based Active } \\
\text { Contour Model }\end{array}$ & {$[8]$} & 2016 & $\mathrm{BC}$ & Nuclei detection & $\begin{array}{c}\text { There is no performance } \\
\text { evaluation for } \\
\text { classification }\end{array}$ \\
\hline $\begin{array}{l}\text { Luminal and } \\
\text { Architecture based } \\
\text { classification }\end{array}$ & {$[35]$} & 2016 & $\mathrm{CaP}$ & Nuclei detection & $\begin{array}{l}97.6 \% \text { accuracy while } \\
\text { segmentation }\end{array}$ \\
\hline $\begin{array}{c}\text { Energy minimization } \\
\text { using graph cut }\end{array}$ & [22] & 2016 & FL & $\begin{array}{l}\text { CB \& non-CB cells } \\
\text { classification }\end{array}$ & $94.56 \%$ accuracy achieve \\
\hline
\end{tabular}




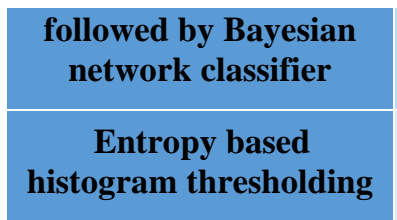

\begin{tabular}{c|c|c|c}
\hline [41] & 2017 & FL & $\begin{array}{c}\text { Segmentation of CD3+ \& } \\
\text { CD3- T cells }\end{array}$
\end{tabular}

Sensitivity and specificity measure $90.97 \%$ \& $88.38 \%$ respectively

Table 1: describes the state of art CAD technologies used in Histopathology. Here results of various segmentation techniques and classification accuracy upon a different set of images pertaining to Cap, BC, FL, and NB are presented.

\section{Discussion and future directions}

Based on the review of the literature, it is concluded that the quantitative analysis from CAD System is very useful in decision making policies. It is successfully applied and benefitted in the diagnosis and in the classification of tissue (as FL, CaP, NB, BC) associated with various grades. The classification is often done by extracting quality parameters like nuclei, Karyorrhectic, background, glands, Centro blast, follicular region, prostate region and the extra-cellular area from the histological section of H\&E Stained images. Various methodologies have been proposed to extract these quality parameters and keep visual difference based on textural heterogeneity $[6,7,8$, $10,11,14,16,19,34,36]$. Several morphological features $[8,12,17,20,22,32,33]$ and their combination with textural feature $[5,10]$, as well as cell graph-based features $[17,22]$, Otsu-thresholding [18] have been introduced to form a feature space vector. This feature vector is often identified in lower dimension using first principle component analysis $[3,7,8,11]$, which is responsible for calculating the main mode of variation in data. Reduced feature vector is then fed into various linear and non-linear classifiers (supervised clustering [17], non-supervised clustering [6], LDA followed by BPE [7, 8, 18, 22],Laplacian Eigen map classifiers [19], K-nearest neighbor [21], neuro-fuzzy inference system [20], boosted multi-resolution [31], rule-based classifiers [19]) to classify these histology into respective grades. Using these approaches classification accuracy ranges from $80 \%$ to $95 \%$ for FL sample, $90 \%$ to $97.6 \%$ for prostate samples, $90 \pm 5 \%$ for breast histology and finally $88.4 \%$ for the neuroblastoma tissue sample. But this level of performance accuracy may not be enough in clinical application and leads to future directions;

- Due to different stain manufacturers and inconsistent biopsy staining and nonstandard imaging can cause color variation in histopathological images [40,42].

- The limited number of datasets leads to inappropriate efficiency of the proposed system in addition to that two different types of stained images (H\&E or IHC) limits the robust performance of segmentation.

- Another motivation is to standardize the evaluation metrics, including accuracy. As M. Oger [15] uses specificity and sensitivity rather Zijdenbos similarity index in [11].

- The higher rate of false positive is still a challenge for FL cases [9].

Over a few decades, a lot of segmentation and classification techniques have been introduced, every researcher has the goal to classify the histopathological images into their respective grades. The essential quality parameter, which can represent these histopathological images, is usually a pre-requisite regarding the classification and state of disease. Other important parameters can be a choice of the classifier, which can deal with large and highly dense datasets. So, this study shows few classifiers and their significance regard to disease grading. Classification accuracy with difference classifier and the quality metrics extracted from histopathological images is shown in Table 1.

In the case of FL, histopathological grading is based on the number of large malignant cells named as CB. It is inferred from the analysis that the demarcation of $\mathrm{CB}$ can improvise the classification accuracy of grades. Moreover, merging of two regions should be minimized in order to compute the disease grading with the desired accuracy. Similarly, NB counting the karyorrhectic cell along with differentiated architecture of nodular pattern leads segmentation accuracy $88.4 \%$. On the other hand, prostate histology, which is solely based on morphological pattern, better computer-assisted view angle color descriptor based meaningful representation leads to max $97.6 \%$ accuracy for segmentation. In case of breast histology, proper segmentation of glands and their representation into biopsy using local binary pattern and local phase quantization, and subsequent classification with SVM classifier leads to more precise segmentation accuracy of $90 \%$.

\section{Reference}

[1] M. N. Gurcan, L. Boucheron, A. Can, A. Madabhushi, N. Rajpoot and B. Yener, "Histopathology Image Analysis: A Review" IEEE Rev. Biomedical Engineering, vol. 2, pp.147-171, (2009). https://doi.org/10.1109/RBME.2009.2034865

[2] L. R. Teras, Carol E. DeSantis, James R. Cerhan, Lindsay M. Mortan, A. Jemal, Christopher R. Flowers, "2016 US Lymphoid Malignancy Statistics by World Health Organization Subtypes", CA: Cancer, Vol. 66, no. 6, (2016). https://doi.org/10.3322/caac.21357

[3] O. Sertel, G. Lozanski, A. Shana'ah, and M. N. Gurcan, "Computer-aided Detection of Centro blast for Follicular Lymphoma Grading using Adaptive Likelihood based Cell Segmentation " IEEE Trans Biomed Engineering pp. 2613-2616, (2010). https://doi.org/10.1109/TBME.2010.2055058

[4] S. Swerdlow, E. Campo, N. Harris, E. Jaffe, S. Pileri, Stein, H. Thiele, and J. Vardiman, "WHO classification of tumors of hematopoietic and 
lymphoid tissues," vol. 2, World Health Organization, Lyon, France, fourth ed. 117(19): 5019-5032 (2008).10.1182/blood-2011-01-293050.

[5] J. Kong, O. Sertel, A. Gewirtz, A. Shana'ah, F. Racke, J. Zhao, K. Boyer, U. Catalyurek, M. N. Gurcan, G. Lozanski, "Development of computer based system to aid pathologists in histological grading of follicular lymphomas", GA. American Society of Histology (2007). https://doi.org/10.1182/blood.V110.11.3318.3318

[6] O. Sertel, J. Kong, G. Lozanski, U. Catalyurek, J. H. Saltz, Metin N. Gurcan, "Computerized microscopic image analysis of follicular lymphoma", SPIE vol. 6915, Medical Imaging (2008). https://doi.org/10.1117/12.770936

[7] O. Sertel, J. Kong, U. Catalyurek, G. Lozanski, A Shanaah, J. H. Saltz, M. N. Gurcan, "Texture classification using nonlinear color quantization: Application to histopathological image analysis" IEEE ICASSP'08; Las Vegas, NV (2008). https://doi.org/10.1109/ICASSP.2008.4517680

[8] O. Sertel, J. Kong, U. Catalyurek, G. Lozanski, Joel H. Saltz, Metin N. Gurcan, "Histopathological Image Analysis Using Model-Based Intermediate Representations and Color Texture: Follicular Lymphoma Grading”, Journal Signal Process System, vol. 55, pp.169-183 (2009). https://doi.org/10.1007/s11265-008-0201-y

[9] O. Sertel, U. Catalyurek, G. Lozanski, A. Shanaah, Metin N. Gurcan, “An Image Analysis Approach for Detecting Malignant Cells in Digitized H\&E-stained Histology Images of Follicular Lymphoma" International Conference on Pattern Recognition (2010). https://doi.org/10.1109/ICPR.2010.76

[10] S. Samsi, G. Lozanski, A. Shana'ah, M. N. Gurcan, "Detection of Follicles from IHC Stained Slide of Follicular lymphoma Using Iterative Watershed", IEEE transaction Biomedical Eng. pp. 2609-2612 Oct. (2010). https://doi.org/10.1109/TBME.2010.2058111

[11] K. Belkacem-Boussaid, M. Pennell, G. Lozanski, A. Shana'ah, and M. Gurcan, "Computer-aided classification of centroblast cells in follicular lymphoma", Anal. Quant. Cytol. Histol., vol.32 no. 5, pp. 254-260 (2010).

PMC ID: [PMC3078581].

K. Belkacem-Boussaid, S. Samsi, G. Lozanski, M.N. Gurcan, "Automatic detection of follicular regions in H\&E images using iterative shape index", Computerized Medical Imaging and Graphics $35 \mathrm{pp}$. 592-602, (2011). https://doi.org/10.1016/ j.compmedimag.2011.03.001

[12] T. F. Chan, Vese LA. Active contours without edges. IEEE Transaction Image Processing, vol. 10, (2001). https://doi.org/10.1109/83.902291

[13] H. Kong, M.N. Gurcan, and K. Belkacem-Boussaid, "Partitioning Histopathological Images: An Integrated Framework for Supervised Color-Texture Segmentation and Cell Splitting" IEEE Transactions On Medical Imaging, Vol. 30, No. 9, (2011).
https://doi.org/10.1109/TMI.2011.2141674

[14] M. Oger, Philippe Belhomme, Metin N. Gurcan, “A general framework for the segmentation of follicular lymphoma virtual slides" Computerized Medical Imaging and Graphics vol. 36, pp. 442-451 (2012). https://doi.org/10.1016/j.compmedimag.2012.05.00 3

[15] S. Samsi, Ashok K. Krishnamurthy, Metin N. Gurcan, "An efficient computational framework for the analysis of whole slide images: Application to follicular lymphoma immunohistochemistry", Journal of Computational Science vol. 3, pp. 269279 (2012). https://doi.org/10.1016/j.jocs.2012.01.009

[16] B. Oztan, H. Kong, M. N. Gurcan, \& B. Yener, "Follicular Lymphoma Grading using Cell-Graphs and Multi-Scale Feature Analysis", Medical Imaging, Proc. of SPIE Vol. 8315 (2012). https://doi.org/10.1117/12.911360

[17] E. Michail, Evgenios N. Kornaropoulos, Kosmas Dimitropoulos, Nikos Grammalidis, Triantafyllia Koletsa, Ioannis Kostopoulos, "Detection of Centro blasts in H\&E Stained Images of Follicular Lymphoma" 2014 IEEE 22nd Signal Processing and Communications Applications Conference 23192322 (2014) https://doi.org/10.1109/SIU.2014.6830728

[18] E. N. Kornaropoulos, M Khalid Khan Niazi, Gerard Lozanski, and Metin N. Gurcan, "Histopathological image analysis for centroblasts classification through dimensionality reduction approaches", Cytometry Analysis, vol. 85, no.3: 242-255 (2014). https://doi.org/10.1002/cyto.a.22432

[19] K. Dimitropoulos, E. Michail, T. Koletsa, I. Kostopoulos, N. Grammalidis, "Using adaptive neuro-fuzzy inference systems for the detection of centroblasts in microscopic images of follicular lymphoma”, Signal, Image Video Process, 8 (1), pp. 33-40, (2014). https://doi.org/10.1007/s11760-014-0688-6

[20] M. F. A. Fauzi, M. Pennell, B. Sahiner, W. Chen, A. Shana'ah, J. Hemminger, A. Gru, H Kurt, M. Losos, A. Joehlin-Price, C. Kavran, S. M. Smith, N. Nowacki, S. Mansor, G. Lozanski and Metin N. Gurcan, "Classification of follicular lymphoma: the effect of computer aid on pathologists grading", BMC Medical Informatics and Decision Making, vol. 15 (2015). https://doi.org/10.1186/s12911-015-0235-6

[21] K. Dimitropoulos, P. Barmpoutis, T. Koletsa, I. Kostopoulos, N. Grammalidis, "Automated detection and classification of nuclei in pax 5 and H\&E-stained tissue sections of follicular lymphoma" Signal, Image Video Process, vol.11, no. 1, pp. 145153(2016). https://doi.org/10.1007/s11760-016-0913-6

[22] O. Sertel, J. Kong, H. Shimada, U.V. Catalyurek, J.H. Saltz, \&M.N. Gurcan, "Computer aided Prognosis of Neuroblastoma on Whole-slide Images: Classification of Stromal Development", Pattern Recognit. vol. 42, no. 6, pp. 1093-1103, (2009). 
https://doi.org/10.1016/j.patcog.2008.08.027

[23] LA. Teot, RSA. Khayat, S. Qualman, G. Reaman, D. Parham, "The problem and promise of central pathology review: Development of a standardized procedure for the children's oncology group", Pediatric and Developmental Pathology, pp. 199207, (2007).

https://doi.org/10.2350/06-06-0121.1

[24] D. Gleason, "Classification of prostatic carcinomas", cancer chemother Rep. pp. 125-128, (1966). [PubMed ID: 5948714].

[25] C. R. King, JP. Long, "prostate biopsy grading error: a sampling problem?", International Journal cancer, (2000). [Pubmed ID: 11180135].

[26] H. J. Bloom, W. Richardson, "Histological grading and prognosis in breast cancer; A study of 1409 cases of which 359 have been followed for 15 years" British Journal of Cancer, Vol. 11 no. 3, pp. 359377, (1957).

https://doi.org/10.1038/bjc. 1957.43

[27] CW. Elston, IO. Ellis, "Pathological prognostic factors in breast cancer. I. The value of histological grade in breast cancer: experience from a large study with long-term follow-up". Histopathology, vol. 19: pp. 403-410, (1991). https://doi.org/10.1111/j.1365-2559.1991.tb00229.x

[28] A. V. Ivshina, J. George, O. Senko, B. Mow, T. C. Putti, J. Smeds, T. Lindahl, Y. Pawitan, P. Hall, H. Nordgren, J. E.L. Wong, E. T. Liu, J. Bergh, V. A. Kuznetsov and L. D. Miller, "Genetic Reclassification of Histologic Grade Delineates New Clinical Subtypes of Breast Cancer" Cancer research, vol. 21, (2006). https://doi.org/10.1158/0008-5472.CAN-05-4414

[29] Bibbo M, Kim DH, Pfeifer T, Dytch HE, GaleraDavidson H, Bartels PH. "Histometric features for the grading of prostatic carcinoma", Anal Quant Cytol. Histol. vol. 13, pp. 61-68 (1991). [PMID: 2025375].

[30] S. Doyle, M. Feldman, J. Tomaszewski, \& A. Madabhushi, "A Boosted Bayesian Multiresolution Classifier for Prostate Cancer Detection from Digitized Needle Biopsies", IEEE trans. on biomedical eng. vol. 59, no. 5, (2012). https://doi.org/10.1109/TBME.2010.2053540

[31] S. Ali \& A. Madabhushi “An Integrated Region-, Boundary-, Shape-Based Active Contour for Multiple Object Overlap Resolution in Histological Imagery" IEEE Transaction on Medical Imaging vol. 31 , no. 7 , pp. 1448-1460, (2012).

https://doi.org/10.1109/TMI.2012.2190089

[32] R. Sparks, A. Madabhushi, "Statistical shape model for manifold regularization: Gleason grading of prostate histology", Computer Vision and Image Understanding, pp.1138-1146, (2013).

https://doi.org/10.1016/j.cviu.2012.11.011.

[33] Safa'a N. Al-Haj Saleh, Moh'd B. Al-Zoubi, "Histopathological Prostate Tissue Glands Segmentation for Automated Diagnosis", 2013 IEEE Jordan Conference on Applied Electrical Engineering and Computing Technologies, (2013).
https://doi.org/10.1109/AEECT.2013.6716471.

[34] M. K. Khan Niazi, K. Yao, D. L Zynger, S. K Clinton, J. Chen, M. Koyutürk, T. La. Framboise, M. Gurcan,

"Visually Meaningful Histopathological Features for Automatic Grading of Prostate Cancer", IEEE Journal of Biomedical and Health Informatics, pp. 2168-2194, (2016). doi: 10.1109/JBHI.2016.2565515.

[35] V. Ojansivu, N. Linder, E. Rahtu, M. Pietikainen, M. Lundin, H. Joensuu, J. Lundin, "Automated classification of breast cancer morphology in histopathological images", Diagnostic Pathology, (2013).

https://doi.org/10.1186/1746-1596-8-S1-S29

[36] M. Paramanandam, M. O'Byrne, B. Ghosh, J. J. Mammen, M. T. Manipadam, R. Thamburaj, V. Pakrashi, "Automated Segmentation of Nuclei in Breast cancer Histopathology Images", PLOS ONE (2016). https://doi.org/10.1371/journal.pone.0162053

[37] G. Anneke, B. Bouwer, G. W. Imhoff, R. Boonstra, E. Haralambieva, A. Berg, B. Jong, "Follicular Lymphoma grade 3B includes 3 cytogenetically defined subgroups with primary $\mathrm{t}(14 ; 18), 3 \mathrm{q} 27$, or other translations: $\mathrm{t}(14 ; 18)$ and $3 \mathrm{q} 27$ are mutually exclusive" blood journal hematology library, Feb. (2013) https://doi.org/10.1182/blood.V101.3.1149

[38] Robert Kridel, Laurie H. Sehn, Randy D. Gascoyne, "Predicting and preventing Transformation of Follicular Lymphoma", Blood Journal ed .1. (2017). https://doi.org/10.1182/blood-2017-05-786178

[39] A. Vahadane, T. Peng, A. Sethi, S. Albarqouni, L. Wang, M. Baust, 'Structure preserving color normalization and sparse stain separation for histological images". IEEE Trans. Med, Imaging; vol. 35 , no. 8 , pp. 1962-1971 (2016). https://doi.org/10.1109/TMI.2016.2529665

[40] F. S. Abas, A. Shana'ah, B. Christian, R. Hasserjian, A. Louissaint, M. Pennell, B. Sahiner, W. Chen, M. K. K. Niazi, G. Lozanski, M. Gurcan, "Computerassisted quantification of CD3 + T cells in follicular lymphoma”, International Society for Advancement of Cytometry, vol. 91, no. 6, pp. 609-621, (2017). https://doi.org/10.1002/cyto.a.23049

[41] A. Thaína, T. Azevedo, A. Leandro, C. Neves, Z. Marcelo, "Segmentation methods of H\&E-stained histological images of lymphoma: A review," Informatics in Medicine, 35-43, (2017). https://doi.org/10.1016/j.imu.2017.05.009 